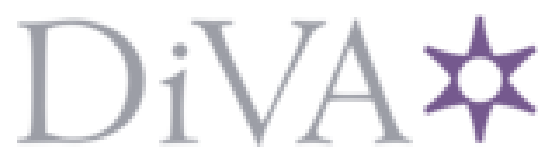

http://www.diva-portal.org

\title{
Postprint
}

This is the accepted version of a paper presented at 2013 IEEE International Symposium on Information Theory, ISIT 2013; Istanbul, Turkey, 7-12 July 2013.

Citation for the original published paper:

Timo, R., Oechtering, T J., Wigger, M. (2013)

Successive refinement with conditionally less noisy side information.

In: IEEE International Symposium on Information Theory: Proceedings (pp. 2985-2989).

IEEE International Symposium on Information Theory - Proceedings

http://dx.doi.org/10.1109/ISIT.2013.6620773

N.B. When citing this work, cite the original published paper.

Permanent link to this version:

http://urn.kb.se/resolve?urn=urn:nbn:se:kth:diva-139143 


\section{Successive Refinement with Conditionally Less Noisy Side Information}

\author{
Roy Timo \\ Institute for Telecommunications Research \\ University of South Australia \\ roy.timo@unisa.edu.au
}

\author{
Tobias J. Oechtering \\ ACCESS Linnaeus Center \\ KTH Royal Institute of Technology \\ oech@kth.se
}

\author{
Michèle Wigger \\ Comm. and Electr. Department \\ Telecom ParisTech \\ michele.wigger@telecom-paristech.fr
}

\begin{abstract}
We consider the successive refinement of information problem with decoder side information. The rate-distortion region is unknown in general; Steinberg \& Merhav and Tian \& Diggavi solved it in the special case of degraded side information. We extend this special case to a new setup, conditionally less noisy side information, and we give a single-letter solution when one distortion function is deterministic.
\end{abstract}

\section{BACKGROUND \& MOTIVATION}

The successive refinement of information problem models scenarios in which data is compressed and decompressed in stages. For example, suppose that a video file is broadcast to many users with different fidelity requirements: Some users have large screens and require high fidelity, while others have small screens and require low fidelity. It is advantageous in such situations to first broadcast low fidelity video to all users, and later send refinements to those requiring high fidelity.

Let us briefly recall the basic problem setup for a discrete memoryless source. The source emits a string of $n$ independent and identically distributed (i.i.d.) random variables $\boldsymbol{X}=X_{1}, X_{2}, \ldots, X_{n}$. An encoder maps $\boldsymbol{X}$ to an initial description (bit stream) at a relatively low rate $R_{1}$, from which a decoder reconstructs a coarse approximation $\hat{\boldsymbol{X}}_{1}$ of $\boldsymbol{X}$. The encoder then generates a second description at rate $R_{2}$, and another decoder reconstructs a fine approximation $\hat{\boldsymbol{X}}_{2}$ using both descriptions - the average distortion of $\hat{\boldsymbol{X}}_{2}$ being less than that of $\hat{\boldsymbol{X}}_{\mathbf{1}}$.

An interesting information-theoretic problem is to determine those rates at which it is theoretically possible to compress $\boldsymbol{X}$. More formally, a rate-distortion (RD) tuple $\left(R_{1}, R_{2}, D_{1}, D_{2}\right)$ is said to be achievable if there exists a compression scheme with refinement rates $R_{1}$ and $R_{2}$ such that the reconstructions $\hat{\boldsymbol{X}}_{1}$ and $\hat{\boldsymbol{X}}_{2}$ have average distortions $D_{1}$ and $D_{2}$ respectively. The $R D$ region is the set of all achievable RD tuples, and the problem is to find a computable expression [1, p. 262] thereof.

Another interesting problem is to determine when successive compression incurs no rate penalty, relative to an optimal source code for each user operating in isolation. Specifically, a source is said to be successively refinable if for every pair of distortions $\left(D_{1}, D_{2}\right)$ it is possible to reliably operate at rates

R. Timo is supported by the Australian Research Council Discovery Grant DP120102123. M. Wigger is partly supported by the city of Paris under the programme "Emergences." corresponding to Shannon's RD function $R_{X}(\cdot)$ [2]; that is, $R_{1}=R_{X}\left(D_{1}\right)$ and $R_{1}+R_{2}=R_{X}\left(D_{2}\right)$.

A plethora of work has been devoted to the aforementioned problems. For example, studies of the RD region date back to Gray and Wyner's seminal paper [3]. Koshelev [4], Equitz and Cover [2] established necessary and sufficient conditions for a source to be successively refinable. Lastras and Berger [5] showed that all memoryless sources with squared error distortion are nearly successively refinable. Successive refinement is a special case of multiple-descriptions coding [6], see El Gamal and Kim [7, Sec. 13.5] for a textbook treatment.

In this paper, we wish to determine the RD region when the decoders have side information (prior knowledge about the source). For example, in video compression $\boldsymbol{X}$ can be the current video frame and the side information can be the previous frames reconstructed by the decoders. Formally, we assume a Wyner and $\mathrm{Ziv}[8]$ setup for the side information: A discrete memoryless source emits an i.i.d. string $\left(\boldsymbol{X}, \boldsymbol{Y}_{\mathbf{1}}, \boldsymbol{Y}_{\mathbf{2}}\right)=\left(X_{1}\right.$, $\left.Y_{1,1}, Y_{2,1}\right),\left(X_{2}, Y_{1,2}, Y_{2,2}\right), \ldots,\left(X_{n}, Y_{1, n}, Y_{2, n}\right)$. Here $\boldsymbol{X}$ is the source to be compressed, and $\boldsymbol{Y}_{\mathbf{1}}$ and $\boldsymbol{Y}_{\mathbf{2}}$ serve as side information about $\boldsymbol{X}$ at the first and second decoders respectively. Neither $\boldsymbol{Y}_{\mathbf{1}}$ nor $\boldsymbol{Y}_{\mathbf{2}}$ is known to the encoder.

The first such study was undertaken by Steinberg and Merhav [9] for degraded side information; that is, assuming $X \multimap-Y_{2} \multimap Y_{1}$ forms a Markov chain. Tian and Diggavi [10] extended the setup, approach and results of [9] from two stages to multiple stages. We extend [9] and [10] to the "conditionally less nosy" setup defined next. The definition is motivated by similar literature on degraded and less noisy broadcast channels [11].

Let $L$ be an auxiliary random variable jointly distributed with $\left(X, Y_{1}, Y_{2}\right)$. We say that $Y_{2}$ is conditionally less noisy than $Y_{1}$ given $L$, abbreviated as $\left(Y_{2} \succeq Y_{1} \mid L\right)$, if

$$
I\left(W ; Y_{2} \mid L\right) \geq I\left(W ; Y_{1} \mid L\right)
$$

holds for every auxiliary random variable $W$ such that $W-\circ$ $(X, L) \multimap\left(Y_{1}, Y_{2}\right)$ forms a Markov chain.

The above definition generalises that used in our preliminary work [13] on a lossless special case of successive refinement. The next proposition shows that degraded is a special case of conditionally less noisy. It can also be shown that the reverse implication is not true: conditionally less noisy does not imply degraded. Proofs of both assertions are given in [12]. 
Proposition 1: If $L \multimap X \multimap\left(Y_{1}, Y_{2}\right)$ forms a Markov chain and the side information is degraded, then $\left(Y_{2} \succeq Y_{1} \mid L\right)$.

The main result of the paper (Theorem/2 in Section II) gives a single-letter characterisation of the RD region when one distortion function is deterministic and the side information is conditionally less noisy.

An important special case of successive refinement with side information is the Kaspi / Heegard-Berger problem [13]-[16]. Here, the encoder only sends one description about $\boldsymbol{X}$ to both decoders, and the problem is to determine the smallest rate at which given distortions can be achieved. Our results for successive-refinement automatically carry over to this setup.

Notation: All random variables are discrete and finite and written in uppercase, e.g., $X$. The alphabet of a random variable is written in matching calligraphic font, e.g. $\mathcal{X}$ is the alphabet of $X$. The $n$-fold Cartesian product of an alphabet is denoted by boldface font, e.g. $\mathcal{X}$ is the $n$-fold product of $\mathcal{X}$.

\section{Formal Problem Statement and Main Result}

Let $\left(X, Y_{1}, Y_{2}\right)$ have a joint distribution $P_{X Y_{1} Y_{2}}$ on $\mathcal{X} \times$ $\mathcal{Y}_{1} \times \mathcal{Y}_{2}$, and let $\left(\boldsymbol{X}, \boldsymbol{Y}_{\mathbf{1}}, \boldsymbol{Y}_{\mathbf{2}}\right)$ be a string of $n$ i.i.d. copies of $\left(X, Y_{1}, Y_{2}\right)$.

A successive-refinement $n$-block code consists of three (possibly stochastic) maps, $f, g_{1}$, and $g_{2}$. The first map $f$ defines an encoder and takes the form

$$
f: \mathcal{X} \longrightarrow \mathcal{M}_{1} \times \mathcal{M}_{2},
$$

where $\mathcal{M}_{1}$ and $\mathcal{M}_{2}$ are finite message index sets. The other two maps, $g_{1}$ and $g_{2}$, define decoders and take the form

$$
\begin{aligned}
& g_{1}: \mathcal{M}_{1} \times \mathcal{Y}_{1} \longrightarrow \hat{\mathcal{X}}_{1}, \\
& g_{2}: \mathcal{M}_{1} \times \mathcal{M}_{2} \times \mathcal{Y}_{2} \longrightarrow \hat{\mathcal{X}}_{2},
\end{aligned}
$$

where $\hat{\mathcal{X}}_{1}$ and $\hat{\mathcal{X}}_{2}$ are $n$-fold Cartesian products of reconstruction alphabets $\hat{\mathcal{X}}_{1}$ and $\hat{\mathcal{X}}_{2}$. The encoder computes $\left(M_{1}, M_{2}\right):=$ $f(\boldsymbol{X})$. The first index $M_{1}$ is sent to both decoders, and the second index $M_{2}$ is sent only to Decoder 2. Decoders 1 and 2 reconstruct $\hat{\boldsymbol{X}}_{\mathbf{1}}:=g_{1}\left(M_{1}, \boldsymbol{Y}_{\mathbf{1}}\right)$ and $\hat{\boldsymbol{X}}_{\mathbf{2}}:=g_{2}\left(M_{1}, M_{2}, \boldsymbol{Y}_{\mathbf{2}}\right)$ respectively.

The two rates of a code $\left(f, g_{1}, g_{2}\right)$ are defined by

$$
\kappa_{j}:=\frac{1}{n} \log _{2}\left|\mathcal{M}_{j}\right|, \quad j=1,2,
$$

and the two average distortions by

$$
\Delta_{j}:=\mathbb{E} \frac{1}{n} \sum_{i=1}^{n} \delta_{j}\left(X, \hat{X}_{j, i}\right), \quad j=1,2,
$$

where $\delta_{j}: \mathcal{X} \times \hat{\mathcal{X}}_{j} \longrightarrow[0, \infty)$ is a bounded distortion function.

An RD tuple $\left(R_{1}, R_{2}, D_{1}, D_{2}\right)$ is said to be achievable if there exists an $n$-block code $\left(f, g_{1}, g_{2}\right)$ - for some sufficiently large blocklength $n-$ satisfying $R_{1} \geq \kappa_{1}, R_{2} \geq \kappa_{2}, D_{1} \geq$ $\Delta_{1}$ and $D_{2} \geq \Delta_{2}$. The closure of the set of all achievable RD tuples $\left(R_{1}, R_{2}, D_{1}, D_{2}\right)$ is called the $R D$ region $\mathcal{R}$.

If in the above problem description we remove the message index $M_{2}$, we obtain the Kaspi/Heegard-Berger problem [14. Sec. IIV], [15]. It can be easily shown that

$$
R_{\mathrm{HB}}\left(D_{1}, D_{2}\right)=\min \left\{R_{1} \geq 0:\left(R_{1}, 0, D_{1}, D_{2}\right) \in \mathcal{R}\right\}
$$

is the RD function for the Kaspi/Heegard-Berger problem.

Our new results for $\mathcal{R}$ and $R\left(D_{1}, D_{2}\right)$ hold when the first reconstruction is an almost lossless copy of a function of $X$; more specifically, we will require that $D_{1}=0$ and $\delta_{1}$ is deterministic in the following sense.

The distortion function $\delta_{1}$ is said to be a deterministic [6], [17] if there is an alphabet $\tilde{\mathcal{X}}$ with $\hat{\mathcal{X}}_{1}=\tilde{\mathcal{X}}$ and a deterministic map $\psi: \mathcal{X} \longrightarrow \tilde{\mathcal{X}}$ such that

$$
\delta_{1}(x, \hat{x})= \begin{cases}0 & \text { if } \hat{x}=\psi(x) \\ 1 & \text { otherwise. }\end{cases}
$$

We henceforth assume $\delta_{1}$ is deterministic, we let

$$
\tilde{X}:=\psi(X),
$$

and we assume $\delta_{2}$ is arbitrary. Also, we denote the restriction of $\mathcal{R}$ to $D_{1}=0$ by

$$
\left.\mathcal{R}\right|_{D_{1}=0}:=\left\{\left(R_{1}, R_{2}, 0, D_{2}\right) \in \mathcal{R}\right\} .
$$

and the restriction of $R_{\mathrm{HB}}\left(D_{1}, D_{2}\right)$ to $D_{1}=0$ by

$$
R_{\mathrm{HB}}\left(0, D_{2}\right):=\min \left\{R_{1} \geq 0:\left(R_{1}, 0,0, D_{2}\right) \in \mathcal{R}\right\} .
$$

Finally, let us define

$$
S\left(D_{2}\right):=\min _{A} I\left(X ; A \mid \tilde{X}, Y_{2}\right), \quad D_{2} \geq 0,
$$

where the minimisation is over all auxiliary $A$ such that

- $A \multimap-X \multimap Y_{2}$ forms a Markov chain;

- the cardinality of the alphabet of $A$ satisfies $|\mathcal{A}| \leq|\mathcal{X}|+1$;

- there exists a map $\phi_{2}: \mathcal{A} \times \tilde{\mathcal{X}} \times \mathcal{Y}_{2} \longrightarrow \hat{\mathcal{X}}_{2}$ such that $D_{2} \geq \mathbb{E} \delta_{2}\left(X, \phi_{2}\left(A, \tilde{X}, Y_{2}\right)\right)$.

The function $S\left(D_{2}\right)$ is non-increasing, convex and continuous in $D_{2}$, see [8, Thm. A2].

Theorem 2: If $\delta_{1}$ is deterministic, $H\left(\tilde{X} \mid Y_{1}\right) \geq H\left(\tilde{X} \mid Y_{2}\right)$ and $\left(Y_{2} \succeq Y_{1} \mid \tilde{X}\right)$, then $\left.\mathcal{R}\right|_{D_{1}=0}$ is equal to the set of RD tuples $\left(R_{1}, R_{2}, 0, D_{2}\right)$ satisfying

$$
\begin{aligned}
R_{1} & \geq H\left(\tilde{X} \mid Y_{1}\right) \\
R_{1}+R_{2} & \geq H\left(\tilde{X} \mid Y_{1}\right)+S\left(D_{2}\right) .
\end{aligned}
$$

Corollary 2.1: Under the conditions of Theorem 2 we have

$$
R_{\mathrm{HB}}\left(0, D_{2}\right)=H\left(\tilde{X} \mid Y_{1}\right)+S\left(D_{2}\right) .
$$

Theorem 2 is proved in Section III-D ahead. In [12] we extend Theorem 2 to three receivers and additionally present results on Tian and Diggavi's "side-information scalable source coding" setup [17].

\section{Proof of Theorem 2 AND Comparison to DEGRADED SIDE INFORMATION}

\section{A. An Achievable RD Region}

A single-letter achievable inner bound to the RD region $\mathcal{R}$ was derived in [16, Thm. 1]. From this result, we now distil a simpler achievable RD region for deterministic $\delta_{1}$ and $D_{1}=0$.

Let $\mathcal{R}_{\text {in }}$ denote the set of all $\left(R_{1}, R_{2}, 0, D_{2}\right)$ satisfying

$$
\begin{aligned}
R_{1} & \geq H\left(\tilde{X} \mid Y_{1}\right) \\
R_{1}+R_{2} & \geq \max \left\{H\left(\tilde{X} \mid Y_{1}\right), H\left(\tilde{X} \mid Y_{2}\right)\right\}+S\left(D_{2}\right) .
\end{aligned}
$$


Lemma 3 (Achievable): If $\delta_{1}$ is deterministic, then

$$
\left.\mathcal{R}\right|_{D_{1}=0} \supseteq \mathcal{R}_{\text {in }} .
$$

Lemma 3 holds for arbitrary side information. Its proof is based on the following scheme. The encoder first sends $\tilde{\boldsymbol{X}}$ to both decoders with rate close to $\max \left\{H\left(\tilde{X} \mid Y_{1}\right), H\left(\tilde{X} \mid Y_{2}\right)\right\}$; this is possible by, e.g., Sgarro [18]. The encoder then sends a lossy copy of $\boldsymbol{X}$ to decoder 2 at rate $S\left(D_{2}\right)$; this is possible by, e.g., Wyner and Ziv [8].

\section{B. The RD Region for Degraded Side Information}

The side information is said to be degraded if

$$
X \multimap Y_{2} \multimap Y_{1}
$$

forms a Markov chain. Steinberg and Merhav [9] and Tian and Diggavi [10] exploited (7) to derive single-letter expressions for the RD region. The result of [10] is summarised next for deterministic $\delta_{1}$ and $D_{1}=0$.

Theorem 4 (Thm. 1, [10]): If the side information is degraded and $\delta_{1}$ deterministic, then $\left.\mathcal{R}\right|_{D_{1}=0}=\mathcal{R}_{\text {in }}$ and (6) simplifies to

$$
\begin{aligned}
R_{1} & \geq H\left(\tilde{X} \mid Y_{1}\right) \\
R_{1}+R_{2} & \geq H\left(\tilde{X} \mid Y_{1}\right)+S\left(D_{2}\right) .
\end{aligned}
$$

The chain (7) only plays a minor role in Tian and Diggavi's achievability proof for Theorem 4 Indeed, essentially the same random-coding argument can be used to prove Lemma 3 . On the other hand, Tian and Diggavi's converse crucially relies on (7), see, for example, the equalities in [10, Eqn. (56)].

\section{Beyond Degraded Side Information}

We now broaden the scope of Theorem 4 to that of Theorem 2. The main effort required will be to derive a new converse without (7). The next lemma does precisely this, and its proof is the topic of Section IV.

Let $\mathcal{R}_{\text {out }}$ denote the set of all $\left(R_{1}, R_{2}, 0, D_{2}\right)$ satisfying

$$
\begin{aligned}
R_{1} \geq & H\left(\tilde{X} \mid Y_{1}\right) \\
R_{1}+R_{2} \geq & H\left(\tilde{X} \mid Y_{1}\right)+S\left(D_{2}\right) \\
& +\min _{W}\left\{I\left(W ; Y_{2} \mid \tilde{X}\right)-I\left(W ; Y_{1} \mid \tilde{X}\right)\right\},
\end{aligned}
$$

where the minimisation is taken over an auxiliary random variable $W$ such that $|\mathcal{W}| \leq|\mathcal{X}|$ and $W \multimap X \multimap\left(Y_{1}, Y_{2}\right)$.

Lemma 5 (Converse): If $\delta_{1}$ is deterministic, then

$$
\left.\mathcal{R}\right|_{D_{1}=0} \subseteq \mathcal{R}_{\text {out }} .
$$

The proof of Lemma 5 is given later in Section IV

\section{Proof of Theorem 2}

By the assumption in the theorem, $H\left(\tilde{X} \mid Y_{1}\right) \geq H\left(\tilde{X} \mid Y_{2}\right)$, and thus by Lemma 3 the region in Theorem 2 is achievable. It remains to prove the converse. The outer bound in Lemma 5 and the region in Theorem 2 differ only in the sum rate constraints $5 \mathrm{~b}$ ) and $8 \mathrm{~b}$. But when $\left(Y_{2} \succeq Y_{1} \mid \tilde{X}\right)$ then by (1)

$$
\min _{W}\left\{I\left(W ; Y_{2} \mid \tilde{X}\right)-I\left(W ; Y_{1} \mid \tilde{X}\right)\right\}=0,
$$

and $(8 \mathrm{~b})$ coincides with $5 \mathrm{~b}$. This establishes the converse.

Theorem 4 is a special case of Theorem 2 Specifically, note that $X \multimap-Y_{2} \multimap Y_{1}$ implies $\left(Y_{2} \succeq Y_{1} \mid X\right)$ and $H\left(\tilde{X} \mid Y_{1}\right) \geq$ $H\left(\tilde{X} \mid Y_{2}\right)$ by Proposition 1 and the data processing lemma.

As a final remark, we note that our proof of Lemma 5 does not readily generalise to an arbitrary distortion function $\delta_{1}$. An apparent difficulty follows from the use of a Wyner-Ziv style converse argument to construct the $S\left(D_{2}\right)$ term using $\left(\tilde{\boldsymbol{X}}, \boldsymbol{Y}_{\mathbf{2}}\right)$ as i.i.d. decoder side information.

\section{Proof of Lemma 5}

We first state the solution to an entropy-characterisation problem: express the difference of two $n$-letter conditional mutual informations in a single-letter form. This problem and solution will play an important role in the proof of Lemma 5

We will need the following notation: For a string of $n$ random variables $\boldsymbol{A}=A_{1}, A_{2}, \ldots, A_{n}$, let $A_{j}^{k}:=A_{j}, A_{j+1}, \ldots$, $A_{k}$ for $1 \leq j \leq k \leq n$.

\section{A. An Entropy-Characterisation Problem}

Consider a tuple of random variables $\left(R, S_{1}, S_{2}, T, L\right)$ with an arbitrary joint distribution. Let $\left(\boldsymbol{R}, \boldsymbol{S}_{\mathbf{1}}, \boldsymbol{S}_{\mathbf{2}}, \boldsymbol{T}, \boldsymbol{L}\right)$ denote a string of $n$ i.i.d. copies of $\left(R, S_{1}, S_{2}, T, L\right)$. Further, suppose that $J$ is jointly distributed with the $n$-string $\left(\boldsymbol{R}, \boldsymbol{S}_{\mathbf{1}}, \boldsymbol{S}_{\mathbf{2}}, \boldsymbol{T}, \boldsymbol{L}\right)$ and $J \multimap(R, L) \multimap\left(\boldsymbol{S}_{1}, \boldsymbol{S}_{2}, \boldsymbol{T}\right)$ forms a Markov chain. Consider the difference $I\left(J ; \boldsymbol{S}_{\mathbf{2}} \mid \boldsymbol{L}\right)-I\left(J ; \boldsymbol{S}_{\mathbf{1}} \mid \boldsymbol{L}\right)$ of $n$-letter conditional mutual informations. We wish to know whether this difference can be expressed in a single-letter form. The next lemma answers this question in the affirmative; its proof is given in Appendix A

Lemma 6: Let $\left(J, \boldsymbol{R}, \boldsymbol{S}_{\mathbf{1}}, \boldsymbol{S}_{\mathbf{2}}, \boldsymbol{T}, \boldsymbol{L}\right)$ be defined as above. There exists an auxiliary random variable $W$ with alphabet $\mathcal{W}$ such that $|\mathcal{W}| \leq|\mathcal{R}||\mathcal{L}|$,

$$
\begin{aligned}
I\left(J ; \boldsymbol{S}_{\mathbf{2}} \mid \boldsymbol{L}\right)-I\left(J ; \boldsymbol{S}_{\mathbf{1}} \mid \boldsymbol{L}\right) & \\
& =n\left(I\left(W ; S_{2} \mid L\right)-I\left(W ; S_{1} \mid L\right)\right)
\end{aligned}
$$

and $W \multimap(R, L) \multimap\left(S_{1}, S_{2}, T\right)$ forms a Markov chain.

Remark 1: The proof of the lemma may remind the reader of steps applied in the converse to the capacity of less noisy broadcast channels. Notice however, that our Lemma 6 does not apply to channel coding problems where the input and output sequences can have memory and thus are no candidates for our $n$-tuples $\boldsymbol{S}_{1}$ and $\boldsymbol{S}_{2}$. In fact, when the $n$-tuples $\boldsymbol{S}_{1}$ and $\boldsymbol{S}_{2}$ are not i.i.d., then in our lemma, the right-hand side of (10) has the extra term $\sum_{i=1}^{n}\left(I\left(S_{1 i}, L_{i} ; S_{1,1}^{i-1}, L_{1}^{i-1}\right)\right.$ $\left.I\left(S_{2 i} L_{i} ; S_{2, i+1}^{n}, L_{i+1}^{n}\right)\right)$, which can be positive or negative. So, also this extended form of our lemma does not directly lead to a converse for capacity problems.

\section{B. Proof of Lemma 5}

Let $\left(R_{1}, R_{2}, 0, D_{2}\right)$ lie in the RD region $\left.\mathcal{R}\right|_{D_{1}=0}$. Then, for every $\epsilon>0$ the tuple $\left(R_{1}+\epsilon, R_{2}+\epsilon, \epsilon, D_{2}+\epsilon\right)$ is achievable i.e., for sufficiently large $n$ we can find an $n$-block 
code $\left(f, g_{1}, g_{2}\right)$ with $\kappa_{1} \leq R_{1}+\epsilon, \kappa_{2} \leq R_{2}+\epsilon, \Delta_{1} \leq \epsilon$ and $\Delta_{2} \leq D_{2}+\epsilon$. For this $n$-block code, we have

$$
\begin{aligned}
R_{1}+\epsilon & \geq \frac{1}{n} H\left(M_{1}\right) \\
& \geq \frac{1}{n} I\left(\tilde{\boldsymbol{X}} ; M_{1} \mid \boldsymbol{Y}_{\mathbf{1}}\right) \\
& \stackrel{\text { (a) }}{\geq} \frac{1}{n}\left(H\left(\tilde{\boldsymbol{X}} \mid \boldsymbol{Y}_{\mathbf{1}}\right)-n \varepsilon_{1}(n, \epsilon)\right) \\
& \stackrel{\text { (b) }}{=} H\left(\tilde{X} \mid Y_{1}\right)-\varepsilon_{1}(n, \epsilon),
\end{aligned}
$$

where (a) applies Fano's inequality and $\varepsilon_{1}(n, \epsilon)$ can be chosen so that $\varepsilon_{1}(n, \epsilon) \rightarrow 0$ as $\epsilon \rightarrow 0$ (for details see [12]); and (b) follows because the pair $\left(\tilde{\boldsymbol{X}}, \boldsymbol{Y}_{\mathbf{1}}\right)$ is i.i.d. Moreover,

$$
\begin{aligned}
& R_{1}+R_{2}+\epsilon \\
& \geq \frac{1}{n} H\left(M_{1}, M_{2}\right) \\
& \geq \frac{1}{n} I\left(\tilde{\boldsymbol{X}}, \boldsymbol{X} ; M_{1}, M_{2} \mid \boldsymbol{Y}_{\mathbf{1}}\right) \\
&= \frac{1}{n}\left(I\left(\tilde{\boldsymbol{X}} ; M_{1}, M_{2} \mid \boldsymbol{Y}_{\mathbf{1}}\right)+I\left(\boldsymbol{X} ; M_{1}, M_{2} \mid \tilde{\boldsymbol{X}}, \boldsymbol{Y}_{\mathbf{1}}\right)\right) \\
& \stackrel{\text { (a) }}{\geq} \frac{1}{n}\left(H\left(\tilde{\boldsymbol{X}} \mid \boldsymbol{Y}_{\mathbf{1}}\right)-H\left(\tilde{\boldsymbol{X}} \mid \boldsymbol{Y}_{\mathbf{1}}, M_{1}\right)\right. \\
&\left.\quad+I\left(\boldsymbol{X} ; M_{1}, M_{2} \mid \tilde{\boldsymbol{X}}, \boldsymbol{Y}_{\mathbf{1}}\right)\right) \\
& \stackrel{\text { (b) }}{=} \frac{1}{n}\left(n H\left(\tilde{X} \mid Y_{1}\right)-n \varepsilon_{1}(n, \epsilon)+I\left(\boldsymbol{X}_{1} M_{1}, M_{2} \mid \tilde{\boldsymbol{X}}, \boldsymbol{Y}_{\mathbf{2}}\right)\right. \\
&\left.\quad+I\left(\boldsymbol{Y}_{\mathbf{2}} ; M_{1}, M_{2} \mid \tilde{\boldsymbol{X}}\right)-I\left(\boldsymbol{Y}_{\mathbf{1}} ; M_{1}, M_{2} \mid \tilde{\boldsymbol{X}}\right)\right)
\end{aligned}
$$

where (a) holds because conditioning does not increase entropy; (b) applies Fano's inequality as in (11), and it uses that $\left(\tilde{\boldsymbol{X}}, \boldsymbol{Y}_{\mathbf{1}}, \boldsymbol{Y}_{\mathbf{2}}\right)$ is i.i.d. and $\left(M_{1}, M_{2}\right) \multimap(\tilde{\boldsymbol{X}}, \boldsymbol{X}) \multimap\left(\boldsymbol{Y}_{\mathbf{1}}, \boldsymbol{Y}_{\mathbf{2}}\right)$.

Consider the first conditional mutual information on the right hand side of (13). We have

$$
\begin{aligned}
& \frac{1}{n} I\left(\boldsymbol{X} ; M_{1}, M_{2} \mid \tilde{\boldsymbol{X}}, \boldsymbol{Y}_{\mathbf{2}}\right) \\
& \quad \stackrel{\text { (a) }}{\geq} \frac{1}{n} \sum_{i=1}^{n} I\left(X_{i} ; M_{1}, M_{2}, Y_{2,1}^{i-1}, Y_{2, i+1}^{n} \mid \tilde{X}_{i}, Y_{2, i}\right) \\
& \quad \stackrel{\text { (b) }}{=} \frac{1}{n} \sum_{i=1}^{n} I\left(X_{i} ; C_{i} \mid \tilde{X}_{i}, Y_{2, i}\right) \\
& \quad \stackrel{\text { (c) }}{\geq} \sum_{i=1}^{n} S\left(\mathbb{E} \delta_{2}\left(X_{i}, \hat{X}_{2, i}\right)\right) \\
& \quad \stackrel{\text { (d) }}{\geq} S\left(\mathbb{E} \frac{1}{n} \sum_{i=1}^{n} \delta_{2}\left(X_{i}, \hat{X}_{2, i}\right)\right) \\
& \quad \stackrel{\text { (e) }}{\geq} S\left(D_{2}+\epsilon\right) .
\end{aligned}
$$

The reasoning behind each step is as follows: (a) $\left(\boldsymbol{X}, \tilde{\boldsymbol{X}}, \boldsymbol{Y}_{\mathbf{2}}\right)$ is i.i.d.; (b) define $C_{i}:=\left(M_{1}, M_{2}, Y_{2,1}^{i-1}, Y_{2, i+1}^{n}\right)$; (c) from the definition of $S(\cdot)$ and since $\hat{X}_{2, i}$ can be written as a function of $\left(C_{i}, Y_{2, i}\right)$; and (d) and (e) by convexity and monotonicity of $S(\cdot)$. From 13 and $(18$, we obtain

$$
R_{1}+R_{2}+\epsilon
$$

$$
\begin{aligned}
& \geq H\left(\tilde{X} \mid Y_{1}\right)+S\left(D_{2}+\epsilon\right)-\varepsilon_{1}(n, \epsilon) \\
& \quad+\frac{1}{n}\left(I\left(M_{1}, M_{2} ; \boldsymbol{Y}_{\mathbf{2}} \mid \tilde{\boldsymbol{X}}\right)-I\left(M_{1}, M_{2} ; \boldsymbol{Y}_{\mathbf{1}} \mid \tilde{\boldsymbol{X}}\right)\right) .
\end{aligned}
$$

Apply Lemma 6 to (19) with $J=\left(M_{1}, M_{2}\right), R=X$, $S_{1}=Y_{1}, S_{2}=Y_{2}$, and $L=\tilde{X}$ : There exists an auxiliary $W$ satisfying $|\mathcal{W}| \leq|\mathcal{X}|$ and $W \multimap X \multimap-\left(Y_{1}, Y_{2}\right)$ such that

$$
\begin{aligned}
R_{1}+R_{2}+\epsilon \geq H\left(\tilde{X} \mid Y_{1}\right) & +S\left(D_{2}+\epsilon\right)-\varepsilon_{1}(n, \epsilon) \\
& +I\left(W ; Y_{2} \mid \tilde{X}\right)-I\left(W ; Y_{1} \mid \tilde{X}\right)
\end{aligned}
$$

The proof follows now by (12) and (20), by taking the limit $\epsilon \rightarrow 0$ together with the continuity of $S(\cdot)$.

\section{APPENDIX A}

\section{PROOF OF LEMMA 6}

The proof will make use of the following telescoping identity. For any string of arbitrarily distributed random variables, $\left(A_{1}, B_{1}\right),\left(A_{2}, B_{2}\right), \ldots,\left(A_{n}, B_{n}\right)$, we have [19, Sec. G]

$$
\sum_{i=1}^{n} I\left(A_{1}^{i} ; B_{i+1}^{n}\right)=\sum_{i=1}^{n} I\left(A_{1}^{i-1} ; B_{i}^{n}\right),
$$

with the notational convention $I\left(A_{1}^{n} ; B_{n+1}^{n}\right) \triangleq 0$ and $I\left(A_{1}^{-1} ; B_{0}^{n}\right) \triangleq 0$.

We first prove $[10$. Notice that

$$
I\left(J ; \boldsymbol{S}_{2} \mid \boldsymbol{L}\right)-I\left(J ; \boldsymbol{S}_{1} \mid \boldsymbol{L}\right)=I\left(J ; \boldsymbol{S}_{2}, \boldsymbol{L}\right)-I\left(J ; \boldsymbol{S}_{1}, \boldsymbol{L}\right),
$$

by the chain rule for mutual information. Expand the first mutual information term $I\left(J ; \boldsymbol{S}_{\mathbf{2}}, \boldsymbol{L}\right)$ on the right hand side of (22) as follows:

$$
\begin{aligned}
& I\left(J ; \boldsymbol{S}_{\mathbf{2}}, \boldsymbol{L}\right) \stackrel{(\text { a) }}{=} \sum_{i=1}^{n} I\left(J ; S_{2, i}, L_{i} \mid S_{2,1}^{i-1}, L_{1}^{i-1}\right) \\
& \stackrel{\text { (b) }}{=} \sum_{i=1}^{n} I\left(J, S_{2,1}^{i-1}, L_{1}^{i-1} ; S_{2, i}, L_{i}\right) \\
& \stackrel{\text { (c) }}{=} \sum_{i=1}^{n}\left(I\left(J, S_{1, i+1}^{n}, S_{2,1}^{i-1}, L_{1}^{i-1}, L_{i+1}^{n} ; S_{2, i}, L_{i}\right)\right. \\
& \left.-I\left(S_{1, i+1}^{n}, L_{i+1}^{n} ; S_{2, i}, L_{i} \mid J, S_{2,1}^{i-1}, L_{1}^{i-1}\right)\right) \\
& \stackrel{\text { (d) }}{=} \sum_{i=1}^{n}\left(I\left(W_{i} ; S_{2, i}, L_{i}\right)\right. \\
& \left.-I\left(S_{1, i+1}^{n}, L_{i+1}^{n} ; S_{2, i}, L_{i} \mid J, S_{2,1}^{i-1}, L_{1}^{i-1}\right)\right)
\end{aligned}
$$

where (a) and (c) follow from the chain rule for mutual information; (b) exploits the fact that the source is i.i.d. and therefore $H\left(S_{2, i}, L_{i} \mid S_{2,1}^{i-1}, L_{1}^{i-1}\right)=H\left(S_{2, i}, L_{i}\right)$; and, finally, in (d) we define and substitute the random variable

$$
W_{i} \triangleq\left(J, S_{1, i+1}^{n}, S_{2,1}^{i-1}, L_{1}^{i-1}, L_{i+1}^{n}\right) .
$$

Expand the second mutual information term $I\left(J ; \boldsymbol{S}_{\mathbf{1}}, \boldsymbol{L}\right)$ on the right hand side of 22) using the telescoping identity (21):

$I\left(J ; \boldsymbol{S}_{\mathbf{1}}, \boldsymbol{L}\right) \stackrel{(\mathrm{a})}{=} \sum_{i=1}^{n}\left(I\left(J, S_{2,1}^{i-1}, L_{1}^{i-1} ; S_{1, i}^{n}, L_{i}^{n}\right)\right.$ 


$$
\begin{aligned}
&\left.-I\left(J, S_{2,1}^{i}, L_{1}^{i} ; S_{1, i+1}^{n}, L_{i+1}^{n}\right)\right) \\
& \stackrel{\text { (b) }}{=} \sum_{i=1}^{n}\left(I\left(J, S_{2,1}^{i-1}, L_{1}^{i-1} ; S_{1, i}, L_{i} \mid S_{1, i+1}^{n}, L_{i+1}^{n}\right)\right. \\
&\left.-I\left(S_{2, i}, L_{i} ; S_{1, i+1}^{n}, L_{i+1}^{n} \mid J, S_{2,1}^{i-1}, L_{1}^{i-1}\right)\right) \\
& \stackrel{\text { (c) }}{=} \sum_{i=1}^{n}\left(I\left(J, S_{1, i+1}^{n}, S_{2,1}^{i-1}, L_{1}^{i-1}, L_{i+1}^{n} ; S_{1, i}, L_{i}\right)\right.\left.-I\left(S_{2, i}, L_{i} ; S_{1, i+1}^{n}, L_{i+1}^{n} \mid J, S_{2,1}^{i-1}, L_{1}^{i-1}\right)\right) \\
& \stackrel{\text { (d) }}{=} \sum_{i=1}^{n}\left(I\left(W_{i} ; S_{1, i}, L_{i}\right)\right. \\
&\left.\quad-I\left(S_{2, i}, L_{i} ; S_{1, i+1}^{n}, L_{i+1}^{n} \mid J, S_{2,1}^{i-1}, L_{1}^{i-1}\right)\right),
\end{aligned}
$$

where (a) invokes the telescoping identity 210 and the chain rule for mutual information; (b) again uses the chain rule; (c) exploits the i.i.d.-ness of the source; and in (d) we substitute for $W_{i}$. Subtract 25) from 23) to obtain

$$
\begin{aligned}
I\left(J ; \boldsymbol{S}_{\mathbf{2}}, \boldsymbol{L}\right) & -I\left(J ; \boldsymbol{S}_{\mathbf{1}}, \boldsymbol{L}\right) \\
& =\sum_{i=1}^{n} I\left(W_{i} ; S_{2, i}, L_{i}\right)-I\left(W_{i} ; S_{1, i}, L_{i}\right) .
\end{aligned}
$$

We now single-letterize the quantity on the right hand side of 26. To this end, we introduce a time-sharing random variable: let $Q$ be uniform on $\{1,2, \ldots, n\}$ and independent of the tuple $\left(\boldsymbol{R}, \boldsymbol{S}_{1}, \boldsymbol{S}_{\mathbf{2}}, \boldsymbol{T}, \boldsymbol{L}\right)$. Dividing 26 by $n$, we have

$$
\begin{aligned}
& \frac{1}{n}\left(\sum_{i=1}^{n} I\left(W_{i} ; S_{2, i}, L_{i}\right)-I\left(W_{i} ; S_{1, i}, L_{i}\right)\right) \\
& \stackrel{\stackrel{(a)}{=}}{n} \frac{1}{n} \sum_{i=1}^{n}\left(I\left(W_{i} ; S_{2, i}, L_{i} \mid Q=i\right)-I\left(W_{i} ; S_{1, i}, L_{i} \mid Q=i\right)\right) \\
& \stackrel{\text { (b) }}{=} I\left(W_{Q} ; S_{2, Q}, L_{Q} \mid Q\right)-I\left(W_{Q} ; S_{1, Q}, L_{Q} \mid Q\right) \\
& \stackrel{\text { (c) }}{=} I\left(W_{Q}, Q ; S_{2, Q}, L_{Q}\right)-I\left(W_{Q}, Q ; S_{1, Q}, L_{Q}\right) \\
& \quad \stackrel{\text { (d) }}{=} I\left(W ; S_{2}, L\right)-I\left(W ; S_{1}, L\right),
\end{aligned}
$$

where in (a) we use that $Q$ is independent of $\left(S_{1, i}, S_{2, i}, L_{i}\right.$, $\left.W_{i}\right)$; in (b) that $Q$ is uniformly distributed; in (c) that $\left(\boldsymbol{S}_{1}, \boldsymbol{S}_{2}, L\right)$ is i.i.d. and independent of $Q$; and, finally, in (d) we define and substitute

$$
W=\left(W_{Q}, Q\right), S_{1}=S_{1, Q}, S_{2}=S_{2, Q}, \text { and } L=L_{Q} .
$$

From 26) and 27), we have

$$
I\left(J ; \boldsymbol{S}_{\mathbf{2}}, \boldsymbol{L}\right)-I\left(J ; \boldsymbol{S}_{\mathbf{1}}, \boldsymbol{L}\right)=n\left(I\left(W ; S_{2}, L\right)-I\left(W ; S_{1}, L\right)\right) .
$$

We also notice that

$$
W_{i} \multimap\left(R_{i}, L_{i}\right) \multimap\left(S_{1, i}, S_{2, i}, T_{i}\right),
$$

forms a Markov chain for all $i=1,2, \ldots, n$. Each of the $n$ Markov chains in (28) follows from (24), the $n$-letter chain

$$
J \multimap(R, L) \multimap\left(S_{1}, S_{2}, T\right),
$$

and the fact that $\left(\boldsymbol{R}, \boldsymbol{S}_{1}, \boldsymbol{S}_{\mathbf{2}}, \boldsymbol{T}, \boldsymbol{L}\right)$ is i.i.d. Now define

$$
R=R_{Q} \quad \text { and } \quad T=T_{Q} .
$$

Using the independence of $Q$ from $\left(\boldsymbol{R}, \boldsymbol{T}, \boldsymbol{S}_{1}, \boldsymbol{S}_{2}, \boldsymbol{L}\right)$, we have the desired Markov chain

$$
W \multimap(R, L) \multimap\left(S_{1}, S_{2}, T\right) .
$$

The proof of the cardinality bound is omitted.

Some final remarks. A consequence of the identity 21 is the classic Csiszár sum identity [7], Sec. 2.4],

$$
\sum_{i=1}^{n} I\left(A_{i} ; B_{i+1}^{n} \mid A_{1}^{i-1}\right)=\sum_{i=1}^{n} I\left(B_{i} ; A_{1}^{i-1} \mid B_{i+1}^{n}\right) .
$$

The proof of Lemma 6 can be manipulated so as to replace the telescoping sum identity step (25) with a Csiszár sum identity step. We feel, however, that the present proof is cleaner.

\section{REFERENCES}

[1] I. Csiszár and J. Körner, Information Theory: coding theorems for discrete memoryless systems, 2nd ed. Cambridge University Press, 2011.

[2] W. H. R. Equitz and T. Cover, "Successive refinement of information," IEEE Trans. Inform. Theory, vol. 37, no. 2, pp. 269-275, 1991.

[3] R. Gray and A. Wyner, "Source coding for a simple network," Bell Sys. Tech. Journal, vol. 53, no. 9, pp. 1681-1721, 1974.

[4] V. N. Koshelev, "Hierarchical coding of discrete sources," Problemy Peredachi Informatsii, vol. 16, no. 3, pp. 31-49, 1980.

[5] L. Lastras and T. Berger, "All sources are nearly successively refinable," IEEE Trans. Inform. Theory, vol. 47, no. 3, pp. 918-926, 2001.

[6] A. El Gamal and T. Cover, "Achievable rates for multiple descriptions," IEEE Trans. Inform. Theory, vol. 28, no. 6, pp. 851-857, 1982.

[7] A. El Gamal and Y.-H. Kim, Network Information Theory. Cambridge University Press, 2011.

[8] A. Wyner and J. Ziv, "The rate-distortion function for source coding with side information at the decoder," IEEE Trans. Inform. Theory, vol. 22, no. 1, pp. 1-10, 1976.

[9] Y. Steinberg and N. Merhav, "On successive refinement for the WynerZiv problem," IEEE Trans. Inform. Theory, vol. 50, no. 8, pp. 16361654, 2004.

[10] C. Tian and S. Diggavi, "On multistage successive refinement for WynerZiv source coding with degraded side informations," IEEE Trans. Inform. Theory, vol. 53, no. 8, pp. 2946-2960, 2007.

[11] J. Körner and K. Marton, "Comparison of two noisy channels," in Topics in Information Theory, Keszthely, Hungry, 1977.

[12] R. Timo, T. J. Oechtering, and M. Wigger, "Source Coding Problems with Conditionally Less Noisy Side Information," submitted to IEEE Trans. Inform. Theory, Dec. 2012. Available at http://arxiv.org/pdf/1212.2396.

[13] R. Timo, T. J. Oechtering, and M. Wigger, "Source coding with conditionally less noisy side information," in IEEE Information Theory Workshop, Lausanne, Switzerland, 2012.

[14] C. Heegard and T. Berger, "Rate distortion when side information may be absent," IEEE Trans. Inform. Theory, vol. 31, no. 6, pp. 727-734, 1985.

[15] A. H. Kaspi, "Rate-distortion function when side-information may be present at the decoder," IEEE Trans. Inform. Theory, vol. 40, no. 6, pp. 2031-2034, 1994.

[16] R. Timo, T. Chan, and A. Grant, "Rate distortion with side-information at many decoders," IEEE Trans. Inform. Theory, vol. 57, no. 8, pp. 5240-5257, 2011.

[17] C. Tian and S. N. Diggavi, "Side-information scalable source coding," IEEE Trans. Inform. Theory, vol. 54, no. 12, pp. 5591-5608, 2008.

[18] A. Sgarro, "Source coding with side information at several decoders," IEEE Trans. Inform. Theory, vol. 23, no. 2, pp. 179-182, 1977.

[19] G. Kramer, "Teaching IT: an identity for the Gelfand-Pinsker converse," IEEE Inform. Theory Society Newsletter, vol. 61, no. 4, pp. 4-6, 2012.

[20] _ "Topics in multi-user information theory," Found. Trends Commun. and Inform. Theory, vol. 4, no. 45, pp. 265-444, 2008. 